fied two classes of release events. In simple events, all the dopamine released from a given synaptic vesicle-membrane fusion site was measured in a single amperometric peak. In complex events, the dopamine released from a given synaptic vesicle-membrane fusion site was measured as a series of discrete peaks. The authors' interpretation is that a simple event consists of a small synaptic vesicle generating a fusion pore in the presynaptic membrane, partially discharging its contents into the synaptic cleft, and then disconnecting from the membrane (Fig. 1b). A complex event occurs when the fusion pore flickers rapidly between an open and closed form, allowing repeated partial release of vesicle contents (Fig. 1c). Comparison of the amperometric traces from simple and complex events supports this interpretation: the number of dopamine molecules oxidized in a simple event is roughly equivalent to the number of dopamine molecules oxidized in the first subunit of a complex event. Thus, both simple and complex events seem to reflect kiss-andrun exocytosis.

The authors found that small synaptic vesicles in midbrain dopaminergic neurons undergo kiss-and-run exocytosis almost exclusively. Kiss-and-run exocytosis is advantageous because it leads to increased longevity of a synaptic vesicle, thereby decreasing the importance of the relatively slow process of vesicle recycling through the endosomal compartment. The authors suggest that such efficient vesicle use is necessary because of the relatively small number of synaptic vesicles present in these midbrain neurons. Kiss-andrun exocytosis also avoids inefficient use of dopamine at synapses that lack well-defined active zones, as is typical of dopaminergic neurons.

The complex form of kiss-and-run may represent a particularly economical form of exocytosis, which may be advantageous if transmitter-loaded vesicles are in short supply. To test this hypothesis, the researchers exposed the cultured neurons to pharmaco- logical agents affecting the secondary messengers that regulate synaptic vesicle cycling. On addition of a phorbol ester, an agent that increases the number of releasable synaptic vesicles, amperometric traces revealed a relative decrease in the number of complex events from $20 \%$ to $6 \%$. After inhibition of protein kinase $\mathrm{C}$, reducing the number of releasable vesicles, the total number of exocytotic events per stimulus was decreased by $82 \%$, but amperometric traces showed a relative increase from $20 \%$ to $40 \%$ in the number of complex events. Thus complex events appear to be favored when fewer releasable vesicles are available.

If the nature of exocytotic mechanisms is determined by the number of vesicles and the nature of the synapse, comparison of the new data ${ }^{1}$ with similar data collected from cells with large dense-core vesicles ${ }^{8}$ should reveal significant variation. Kiss-and-run occurs in both cases, but there are some notable differences. First, the amperometric trace subunit duration is approximately 200 times shorter in small synaptic vesicles. Second, the fusion pore flickering occurs with a ten-fold increase in frequency in small synaptic vesicles compared to the large dense-core vesicles. Third, the small synaptic vesicles release $25-30 \%$ of their dopamine cargo with each flicker of the fusion pore, whereas the large dense-core vesicles release $<1 \%$ of their dopamine. Clearly, although the same exocytotic mechanism is at work, the fusion pore flickering characteristics are greatly influenced by the size of the vesicle and the function of the cell.

Some questions remain. The new research ${ }^{1}$ suggests that kiss-and-run exocytosis is driven by the need for efficient use of a relatively small number of synaptic vesicles. This hypothesis can be further tested by measuring the relative number of full fusion and kissand-run events at presynaptic terminals in neurons with a larger number of vesicles, and in neurons that use other neurotransmitters. Amperometry can only detect easily oxidized neurotransmitters such as dopamine, so new strategies will need to be developed for other transmitters such as glutamate. Extracellular calcium is central in regulating exocytosis and release probabilities, and it will be fascinating to explore its influence on the characteristics of kiss-and-run and the flickering pore. Ideally, this would entail amperometric measurements and simultaneous calcium imaging. Because the small synaptic vesicles release such a large percentage of their total neurotransmitter concentration with each flicker of the fusion pore, it would also be interesting to manipulate the intravesicular contents to see whether the kiss-and-hold state can be induced in neurons. Large vesicles forced into a kiss-and-hold state through increased extracellular osmotic pressure undergo massive release when returned to isotonic conditions. Will similar manipulations force the small synaptic vesicles from kiss-and-run to full fusion exocytosis? Future work will tell us whether non-dopaminergic neurons also use a nearly exclusive kiss-and-run mechanism of exocytosis and will explore the implications of kiss-and-run vesicle re-use for the synaptic vesicle recycling mechanism.

1. Staal, R.G.W., Mosharov, E.V. \& Sulzer, D. Nat. Neurosci. 7, 341-346 (2004).

2. Heuser, J.E. Q. J. Exp. Physiol. 74, 1051-1069 (1989).

3. Viveros, O.H. in Handbook of Physiology Vol. 6 (eds. Blaschko, A. \& Smith, A.D.) 389-426 (American Physiological Society, Washington, D.C., 1975).

4. Neher, E. \& Marty, A. Proc. Natl. Acad. Sci. USA 79, 6712-6716 (1982).

5. Wightman, R.M. et al. Proc. Natl. Acad. Sci. USA 88, 10754-10758 (1991).

6. Finnegan, J.M. et al. J. Neurochem. 66, 1914-1923 (1996).

7. Steyer, J.A. \& Almers, W. Biophys. J. 76, 2262-2271 (1999).

8. Alvarez de Toledo, G., Fernandez-Chacon, R. \& Fernandez, J.M. Nature 363, 554-558 (1993).

9. Troyer, K.P. \& Wightman, R.M. J. Biol. Chem. 277, 29101-29107 (2002).

10. Amatore, C., Bouret, Y., Travis, E.R. \& Wightman, R.M. Angew. Chem. Int. Ed. Engl. 39, 1952-1955 (2000).

11. Klyachko, V.A. \& Jackson, M.B. Nature 418, 89-92 (2002).

12. Aravanis, A.M., Pyle, J.L., Harata, N.C. \& Tsien, R.W. Neuropharmacology 45, 797-813 (2003).

13. Gandhi, S.P. \& Stevens, C.F. Nature 423, 607-613 (2003).

\title{
Stiffening the spines
}

The ability of dendritic spines to change shape in response to synaptic activity is crucial for synaptic plasticity. This motility is regulated by $\alpha \mathrm{N}$-catenin, report Abe et al. on page 357. Overexpression of $\alpha \mathrm{N}$-catenin (green; red is PSD95) stabilized spines in cultured neurons, reducing turnover and thereby increasing their number. Lack of $\alpha \mathrm{N}$-catenin increased spine motility, even at established synaptic contacts. Spine $\alpha \mathrm{N}$-catenin was regulated by synaptic activity: blocking activity with tetrodotoxin reduced $\alpha \mathrm{N}$-catenin staining (and increased spine motility), whereas blocking inhibitory neurotransmission increased $\alpha \mathrm{N}$-catenin. The catenins link cadherin cell adhesion molecules to the cytoskeleton, so $\alpha \mathrm{N}$-catenin is well placed to regulate spine dynamics.

\section{Annette Markus}

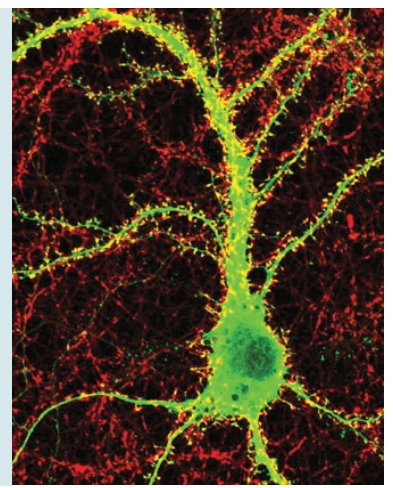

